\title{
Motivational Tuning of Fronto-Subthalamic Connectivity Facilitates Control of Action Impulses
}

\author{
Damian M. Herz, ${ }^{1}$ Mark S. Christensen, ${ }^{1,2,3}$ Norbert Bruggemann, ${ }^{1}$ Oliver J. Hulme, ${ }^{1}$ K. Richard Ridderinkhof, ${ }^{4,5}$ \\ Kristoffer H. Madsen, ${ }^{1,6}$ and Hartwig R. Siebner ${ }^{1,7,8}$ \\ ${ }^{1}$ Danish Research Centre for Magnetic Resonance, Centre for Functional and Diagnostic Imaging and Research, Copenhagen University Hospital Hvidovre, \\ 2650 Hvidovre, Denmark, ${ }^{2}$ Department of Neuroscience and Pharmacology and ${ }^{3}$ Department of Nutrition, Exercise, and Sports, University of Copenhagen, \\ 2200 Copenhagen, Denmark, ${ }^{4}$ Department of Psychology and ${ }^{5}$ Cognitive Science Center Amsterdam, University of Amsterdam, 1018 Amsterdam, The \\ Netherlands, ${ }^{6}$ DTU Informatics, Technical University of Denmark, 2800 Lyngby, Denmark, ${ }^{7}$ Department of Neurology, Copenhagen University Hospital \\ Bispebjerg, 2400 Copenhagen, Denmark, and ${ }^{8}$ Department of Clinical Medicine, Faculty of Health and Medical Sciences, University of Copenhagen, 2200 \\ Copenhagen, Denmark
}

It is critical for survival to quickly respond to environmental stimuli with the most appropriate action. This task becomes most challenging when response tendencies induced by relevant and irrelevant stimulus features are in conflict, and have to be resolved in real time. Inputs from the pre-supplementary motor area (pre-SMA) and inferior frontal gyrus (IFG) to the subthalamic nucleus (STN) are thought to support this function, but the connectivity and causality of these regions in calibrating motor control has not been delineated. In this study, we combined off-line noninvasive brain stimulation and functional magnetic resonance imaging, while young healthy human participants performed a modified version of the Simon task. We show that impairing pre-SMA function by noninvasive brain stimulation improved control over impulsive response tendencies, but only when participants were explicitly rewarded for fast and accurate responses. These effects were mediated by enhanced activation and connectivity of the IFG-STN pathway. These results provide causal evidence for a pivotal role of the IFG-STN pathway during action control. Additionally, they suggest a parallel rather than hierarchical organization of the pre-SMA-STN and IFG-STN pathways, since interruption of pre-SMA function can enhance IFG-STN connectivity and improve control over inappropriate responses.

Key words: fMRI; motor control; pre-SMA; reward; Simon task; TMS

\section{Introduction}

In the human motor system, fast automatic reactions elicited by overlearned stimulus-response rules have the advantage of minimizing metabolic cost, computational complexity, and processing time. Such routine reactions, however, have to be overridden if they are in conflict with a new stimulus-response mapping rule. For instance, when using a computer with different "language settings," some letters on the keyboard are assigned to keys, which are incompatible to the overlearned key-to-letter mapping rule. In this case, automatic tendencies to press keys according to the "old" overlearned spatial mapping rule have to be suppressed to avoid writing mistakes. This is one of many situations in daily life where the brain networks engaged in motor control have to

\footnotetext{
Received Sept. 24, 2013; revised Jan. 8, 2014; accepted Jan. 16, 2014.

Author contributions: D.M.H., M.S.C., K.H.M., and H.R.S. designed research; D.M.H., M.S.C., and N.B. performed research; D.M.H., M.S.C., K.H.M., and H.R.S. analyzed data; D.M.H., M.S.C., N.B., O.J.H., K.R.R., K.H.M., and H.R.S. wrote the paper.

This work was supported by the Danish Medical Research Council (FSS 09-072163) and Lundbeckfonden (Grant of Excellence "ContAct" R59 A5399).

The authors declare no competing financial interests.

Correspondence should be addressed to Damian M. Herz, Danish Research Centre for Magnetic Resonance (DRCMR), Centre for Functional and Diagnostic Imaging and Research, Copenhagen University Hospital Hvidovre, Kettegaard Alle 30, 2650 Hvidovre, Denmark. E-mail: damianh@drcmr.dk.

DOI:10.1523/JNEUROSCI.4081-13.2014

Copyright $\odot 2014$ the authors $\quad 0270-6474 / 14 / 343210-08 \$ 15.00 / 0$
}

solve conflicts between competing response tendencies and to prioritize a specific action according to task requirements.

Recent studies have provided converging evidence that the pre-supplementary motor area (pre-SMA) and inferior frontal gyrus (IFG) play a central but differential role in controlling inappropriate response tendencies via their connections to the subthalamic nucleus (STN) (Ridderinkhof et al., 2011). There is evidence that connectivity in motor control networks is sensitively tuned to the incentives of outcomes, which has been linked to reward-related improvements in motor performance, e.g., increased response speed (Harsay et al., 2011). However, crucial knowledge about causal interactions in the pre-SMA-IFG-STN network and its modulation by motivational incentives is lacking.

Results of previous studies indicate a hierarchical organization of the motor control network, in which pre-SMA detects response conflict and recruits IFG to stop inappropriate actions via its pathway to the STN (Forstmann et al., 2010; Hikosaka and Isoda, 2010; Neubert et al., 2010; Swann et al., 2012). When high response speed is incentivized, such hierarchical models predict an increase in pre-SMA-STN connectivity to control the increased risk of impulsive errors. Thus, disruption of pre-SMA should result in decreased response accuracy when fast responses are rewarded due to a decreased pre-SMA-STN connectivity and impaired recruitment of the IFG-STN pathway. Conversely, it 
has also been suggested that pre-SMA-STN and IFG-STN connections constitute parallel (though interacting) pathways and that the IFG-STN pathway takes over control when the preSMA-STN pathway is insufficient in controlling erroneous responses, e.g., during high response speed (Wiecki and Frank, 2010). Since such parallel models postulate a pivotal role of the IFG-STN pathway during high response speed, they predict an increase in IFG-STN connectivity when fast responses are incentivized. Disruption of pre-SMA activity should result in enhanced IFG-STN connectivity enabling preserved motor control during prospect of reward for fast responses.

To test these predictions, we acquired functional magnetic resonance imaging (fMRI) data, while healthy volunteers performed a Simon task with and without financial incentives for fast and accurate trials (Fig. 1a). Participants performed the task after $1 \mathrm{~Hz}$ repetitive transcranial magnetic stimulation (rTMS) serving to transiently disrupt pre-SMA function and lowintensity sham rTMS as a control condition on two different sessions. This allowed inference on the organization, connectivity, and causality of the pre-SMA-IFG-STN network during motor conflict and its modulation by motivational incentives.

\section{Materials and Methods}

Participants. Fourteen healthy volunteers (7 females; mean age: 23 years \pm 3.9 years) with no history of major diseases including neurological disorders participated in the experiment. All participants were righthanded as assessed by the Edinburgh Handedness Inventory (Oldfield, 1971). The participants were paid 1000 DKK $(\sim \$ 170)$ for participation. Additionally, they could win a monetary reward of a maximum of 500 DKK ( $\sim 85)$ during the experimental sessions. In accordance with the declaration of Helsinki all participants gave their written informed consent to the study, which was approved by the local ethics committee of the University of Copenhagen, Denmark (H-KF-01-131/03).

Experimental task. To probe control over inappropriate response tendencies, we used a modified version of the Simon task (Fig. $1 a$; Simon, 1967). In the task, participants had to press the right or left button of a computer mouse with, respectively, the index or middle finger of their right hand depending on the color of a stimulus, which was presented on either the left or right side of the screen. Thus, the cue had a relevant dimension (color) and an irrelevant dimension (spatial position). Stimuli were labeled as Corresponding when spatial position and color-based response side coincided, and Noncorresponding when spatial position was opposite to the side designated by stimulus color. According to feature integration theory, response conflict is strongest when one stimulus dimension alternates between trials, while the other stimulus dimension stays the same (Hommel et al., 2004). In this case, one has to execute the alternate response, if the relevant stimulus dimension changed between trials, or repeat the response of the previous trial if the irrelevant stimulus dimension changed. Conversely, the response tendency (alternate or same response as previous trial) elicited by the relevant and irrelevant stimulus feature is identical, when both stimulus dimensions stay the same, or both stimulus dimensions change. We refer to the former trials as incompatible, and to the latter trials as compatible trials. In this study we focused on data analysis according to feature integration theory, but we also report results of the analysis according to the classical withintrials conflict (Corresponding and Noncorresponding trials).

Associations between the color of the cue and the appropriate response were counterbalanced across subjects and sessions (real and sham rTMS), but did not change within a session. In $50 \%$ of all trials, red and blue coins were presented instead of circles. In these Prospect-of-reward trials, the level of extrinsic motivation was increased by financially rewarding trials in which participants performed faster than the group average and correctly. No feedback was given during the experiment to avoid interference between neural activity related to prospect of reward and activity related to processing of reward outcomes.

One experimental session consisted of three fMRI runs, which comprised 168 experimental trials each. Each trial started with a central fix- ation cross with a variable duration from $1500-2500 \mathrm{~ms}$. After this fixation display, the respective stimulus was presented for $1500 \mathrm{~ms}$. After eight trials of no-reward stimuli (circles), a central fixation cross was presented for $4000 \mathrm{~ms}$ followed by eight trials of prospect-of-reward stimuli (coins). These small blocks of no-reward and Prospect-of-reward stimuli were alternating throughout each run. A single fMRI run lasted $\sim 13 \mathrm{~min}$. Stimuli were pseudorandomly generated using E-Prime 2.0 (Psychology Software Tools) and a different stimulus order was generated for each run. We restricted stimulus generation by presenting each trial type at an equal probability in each run. We additionally included 24 null trials per run (1 per block), in which only a central fixation cross was presented. Before the rTMS and fMRI session, participants completed a training program of 10 min to familiarize themselves with the stimulusresponse associations.

rTMS. Each participant underwent real and sham rTMS, delivered outside the MRI scanner, on 2 separate days, at least 1 week apart. The order of intervention was counterbalanced across subjects. In each rTMS session, 1800 biphasic stimuli were given over the pre-SMA using a P-Stim 160 stimulator and a figure-eight-shaped coil (double $95 \mathrm{~mm}$; MAG \& More). All participants received $30 \mathrm{~min}$ of $1 \mathrm{~Hz}$ rTMS, which has been shown to decrease cortical excitability at the stimulation site (Siebner and Rothwell, 2003). According to a previous study (Rushworth et al., 2002) the pre-SMA stimulation site was located $4 \mathrm{~cm}$ anterior to electrode position $\mathrm{Cz}$ with the handle pointing from right to left. We chose this site because activations related to response capture during the Simon task are localized in the caudal portions of the pre-SMA (Forstmann et al., 2008). Stimulation intensity was set to $100 \%$ of the individual resting motor threshold (RMT; motor-evoked potential $>50 \mu \mathrm{V}$ in $5 / 10$ consecutive stimuli) for real rTMS and $30 \%$ of individual RMT for sham stimulation. We used frameless stereotaxy (TMS Navigator; Localite) to ensure a constant position of the coil throughout the rTMS session. All participants wore earplugs to protect against TMS-related noise and to diminish differences in noise induced by real and sham rTMS. The time from the end of rTMS to the commencement of fMRI acquisition was $\sim 5 \mathrm{~min}$.

fMRI acquisition and preprocessing. After rTMS, fMRI data were acquired on a $3 \mathrm{~T}$ Verio scanner (Siemens). A scanning session consisted of three runs. In each run 425 functional T2*-echo planar images (EPI; FOV $192 \mathrm{~mm}$, slice thickness $3.5 \mathrm{~mm}$, slice spacing $0.2 \mathrm{~mm}$, TR $1850 \mathrm{~ms}$, TE 26 $\mathrm{ms}, \mathrm{FA} 75^{\circ}, 36$ slices, ascending acquisition order, whole-brain coverage) were acquired. Visual stimuli were projected on a screen and were viewed through a mirror attached to the head coil. The fMRI data were preprocessed using statistical parametric mapping (SPM8; Wellcome Trust Centre for Neuroimaging, London, UK) implemented in MATLAB 7.10.2 (MathWorks). The first three volumes were discarded to allow for T1-equilibrium effects. The remaining images were realigned to the mean EPI image of the time series to correct for small head movements. The resulting images were normalized to a standard EPI template based on the Montreal Neurological Institute (MNI) reference brain using linear and nonlinear transform, resampled to $2 \times 2 \times 2 \mathrm{~mm}$ voxels, and smoothed with an isotropic $8 \mathrm{~mm}$ full-width half-maximum Gaussian kernel, to allow for intersubject anatomical differences and to allow valid statistical inference according to Gaussian random field theory. Additionally, the data were filtered in the temporal domain using a high-pass filter with a frequency of $1 / 128 \mathrm{~Hz}$ to correct for baseline drifts.

Analysis of behavioral data. Mean reaction times (RT) and accuracy rates were used for statistical analyses. In the Simon task, errors typically occur due to impulsive fast responses (Ridderinkhof, 2002). Therefore, accuracy rates were divided into fast and slow responses after a median split. We refrained from dividing responses into more bins, because we had to restrict the number of experimental trials due to the limited duration of rTMS-induced aftereffects (Siebner and Rothwell, 2003). Extreme RT values defined as either anticipatory responses faster than 200 ms or excessively delayed responses slower than 1500 ms were removed from analysis. Main effects of Prospect-of-reward, rTMS, and incompatibility on the independent variable RT were tested in a $2 \times 2 \times 2$ repeated-measures ANOVA. Accuracy rates were tested in a $2 \times 2 \times 2 \times$ 2 ANOVA with the additional factor Response-speed. In case of significant interactions, post hoc comparisons were conducted and corrected 
for multiple comparisons using Bonferronicorrection when applicable. We used Levene's test to ensure equality of distribution of error variance before conducting the ANOVAs.

Analysis of fMRI data. The statistical analysis of fMRI data was performed using the general linear model (GLM). The design matrix was generated with a synthetic hemodynamic response function. Recordings of respiration and cardiac pulsation were included as nuisance covariates in the design matrix (Lund et al., 2006). We also included 24 regressors to remove residual movement artifacts. Residual movement effects were modeled using an expansion of the estimated movement parameters from the rigid body realignment procedure (six parameters). The expansion included the motion parameters, the motion parameters from the previous volume, squared motion parameters, and squared motion parameters from the previous volume (Friston et al., 1996). Trials following an erroneous trial or a null trial were modeled as regressors of no interest. Main effects for Prospect-of-reward (Prospect-ofreward vs no-reward trials), Incompatibility (incompatible vs compatible trials), and rTMS (real vs sham rTMS) were analyzed by computing the respective contrasts on the first level and a consecutive one-sample $t$ test on the second level. Results were thresholded at a cluster-corrected threshold of 0.05 using the familywise error method implemented in SPM.

Based on the results from the behavioral data (see Results), we then conducted fMRI covariance analysis. In covariance-based fMRI analyses individual differences in performance are considered to provide important information for elucidating neural correlates of behavior rather than merely modeling individual differences as error variance (Kosslyn et al., 2002). Contrast maps for the contrast "Prospectof-reward vs no-reward trials" for incompatible trials were computed for each participant in a first-level analysis and entered into a paired samples $t$ test for the sham and real rTMS condition on the second level. Crucially, the reward-related benefit in accuracy on incompatible trials was entered as a covariate in the regression model. It was defined as (Accuracy $_{\text {Reward }}-$ Accuracy $_{\text {Neutral }}$ )/ Accuracy $_{\text {Neutral }}$ based on Harsay et al. (2011).

Based on our a priori hypothesis, we defined IFG and STN contralateral to the moving hand as regions of interest using a $12 \mathrm{~mm}$ sphere. Since IFG activation during motor control has often been reported to be right lateralized (Aron, 2011), we applied the same analysis to the ipsilateral (right) IFG and STN. Coordinates were based on a meta-analysis of IFG activation during response inhibition (IFG coordinates in MNI space: -49 11 4; Rubia et al., 2001) and an anatomical atlas (STN coordinates in MNI space: $-10-15-5$; Lucerna et al., 2002), respectively.

Analysis of effective connectivity. Dynamic causal modeling (DCM) was applied to assess changes in effective connectivity. DCM models the hidden neural dynamics underlying changes in the fMRI signal based on a set of differential equations, comprising an A-matrix (baseline coupling), a B-matrix (contextual modulation of the connections; here, Prospect-ofreward and rTMS), and a C-matrix (direct inputs to the system; here onsets of compatible and incompatible trials; Friston et al., 2003). Based

b
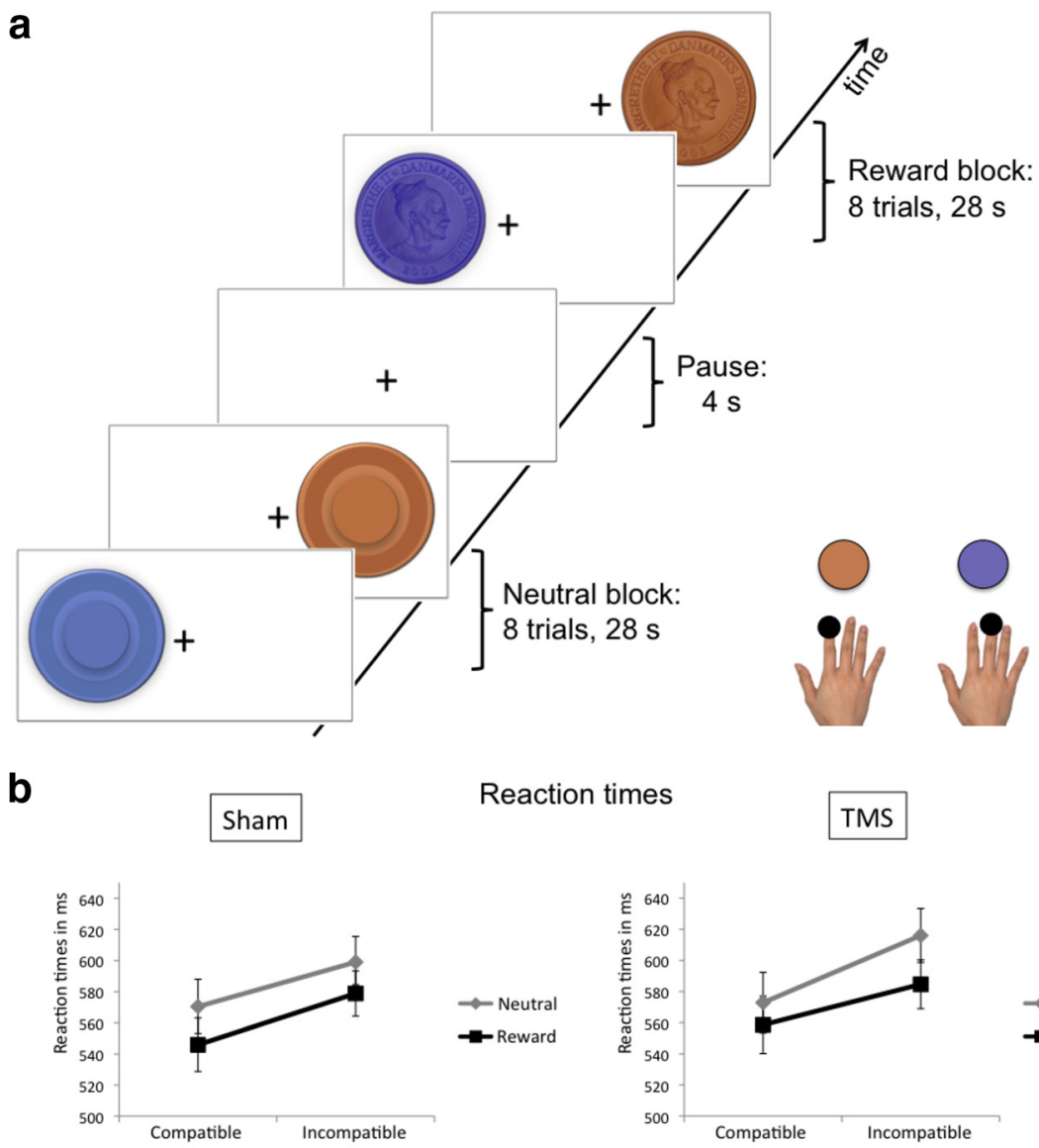

Reaction times

TMS
C

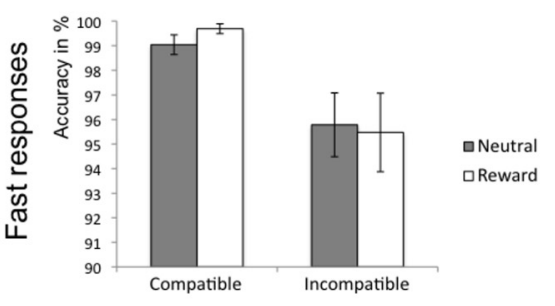

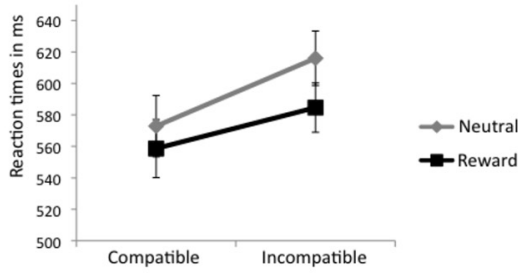

Accuracy rates

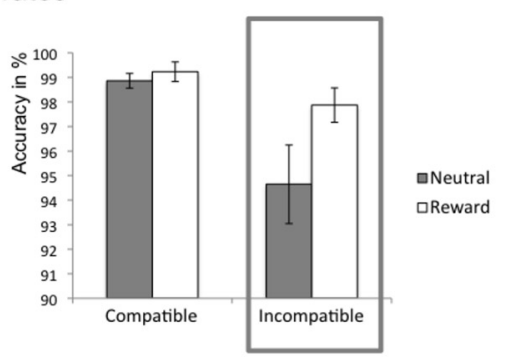

Figure 1. Experimental task and behavioral results. $a$, Experimental task. During fMRl, subjects performed a Simon task, which required subjects to make a correct response while suppressing an inappropriate response. On each trial a cue was presented on either the left or right side of the screen. Depending on the color of the cue, participants had to respond with a button press of the index or middle finger of their right hand. When one cue dimension (e.g., color) alternated between trials, while the other cue dimension (e.g., spatial position) was repeated (incompatible trials), there was an increased risk of impulsive inappropriate responses compared with consecutive trials where both cue dimensions change or stay the same (compatible trials). The level of motivation was manipulated by the presence or absence of a financial incentive. Participants could win a monetary reward in $50 \%$ of the trials, which were indicated by a coin instead of a circle. $\boldsymbol{b}$, Mean RTs after sham (left) and real rTMS (right). Error bars indicate SEM. c, Mean accuracy rates after sham (left) and real rTMS (right) for fast responses. Trials were divided into slow and fast responses after a median split. The gray box shows a selective improvement in accuracy after real rTMS for trials with a high level of response conflict (incompatible trials) and a high level of motivation (rewarding context). Error bars indicate SEM.

on our SPM results and previous neuroimaging studies (Aron et al., 2007; Neubert et al., 2010), we constructed a DCM including the IFG, STN, and pre-SMA in the left hemisphere as a parsimonious neural model for motor control. The coordinates of IFG and STN were based on the local activation peaks from the fMRI covariance analysis. The coordinates for pre-SMA were based on the peak activation during the contrast "sham vs real rTMS" on the second-level fMRI analysis ( $-81052, \mathrm{MNI} ; P_{\text {uncorrected }}<$ 0.005). The fMRI time series were extracted from all voxels within a $4 \mathrm{~mm}$ radius of these coordinates, from each subject, using the first eigenvariate. We refrained from using a bigger sphere or displacement of the 
centers of the spheres due to the small size of the STN. We included a direct connection from both pre-SMA and IFG to the STN (Monakow et al., 1978; Jahfari et al., 2012; Lambert et al., 2012), and a reciprocal connection between IFG and pre-SMA (Johansen-Berg et al., 2004; Aron et al., 2007). Input (C-matrix) was set to pre-SMA, because motor control-related activation of pre-SMA precedes activation of the IFG (Neubert et al., 2010; Swann et al., 2012).

We created and compared several alternative models (Fig. 3a). The critical difference was whether Prospect-of-reward, rTMS, or both modulated corticosubthalamic connectivity and whether the pre-SMA-STN connection, IFG-STN connection, or both were modulated. We also included a null model, which postulated that there was no modulation of neural connectivity. This resulted in 10 different models for each participant. To model rTMS as a modulatory input in the DCM, fMRI data of the sham and real rTMS sessions were concatenated in a new GLM and all models were then fitted to the fMRI data. We used Bayesian model selection (BMS) to detect the model that best explained the data taking into account model complexity (Penny et al., 2004). Differences in free energy $(\Delta \mathrm{F})>5$ indicate very strong evidence for a given model (Friston et al., 2007). Having identified the most likely model, the connectivity parameters (B-matrix) of the best model were extracted to test whether modulation of corticosubthalamic connectivity predicted the observed changes in performance. To specifically assess predictions of variance, which was not already explained by difference in neural activity, we applied hierarchical multiple linear regression analysis. Here, we added the individual B-values, reflecting, respectively, modulation by Prospect-of-reward and rTMS, into a regression model containing the eigenvariates from the covariance-based fMRI analysis (see above, Analysis of fMRI data) as independent variables (predictors). This allowed testing whether predictions of the reward-related benefit in performance after rTMS were significantly improved by including modulation of neural connectivity.

\section{Results}

\section{Performance}

In line with previous work (Hommel et al., 2004), participants needed to slow down their motor response to inhibit the incorrect response tendency evoked by the recent history of the irrelevant stimulus dimension, and to execute the appropriate response (Fig. $1 b$ ). Mean RT ( $\pm \mathrm{SD}$ ) was $595 \mathrm{~ms}$ ( $\pm 55 \mathrm{~ms}$ ) in incompatible trials compared with $562 \mathrm{~ms}( \pm 63) \mathrm{ms}$ in compatible trials, resulting in a significant main effect of Incompatibility: $F_{(1,13)}=21.127, p=0.001$. Of note, this between-trial effect on RT (33 ms) was much more pronounced than the classical within-trial effect of response conflict, i.e., the Simon effect, with RTs being $9 \mathrm{~ms}$ slower in Noncorresponding compared with Corresponding trials $\left(F_{(1,13)}=5.646, p=0.034\right)$.

As expected from the effects of monetary outcomes on response speed (Harsay et al., 2011) prospect of reward had a strong effect on RT, with RT being 23 ms faster in the Prospect-ofreward condition than in the No-reward condition (Main effect of Prospect-of-reward: $567 \pm 55 \mathrm{~ms}$ vs $590 \pm 61 \mathrm{~ms}, F_{(1,13)}=$ $16.65, p=0.001$; Fig. $1 b)$. There was no main effect of rTMS on RT ( $p=0.386)$ or any interactions between Prospect-of-reward, rTMS and response conflict (neither for Incompatibility nor for the Simon effect).

In a next step, we analyzed accuracy rates to assess control over inappropriate responses. When analyzing the classical withintrial conflict, there was no main effect of Correspondence $\left(F_{(1,13)}=0.2, p=0.662\right)$, or interactions of Correspondence with rTMS $\left(F_{(1,13)}=1.479, p=0.246\right)$ or Prospect-of-reward $\left(F_{(1,13)}=1.967, p=0.184\right)$. On the other hand, incompatibility between trials (according to the feature integration approach) had a significant effect on accuracy rates, which were significantly lower in incompatible trials compared with compatible trials (Main effect of Incompatibility: 96.6 vs $99.1 \%, F_{(1,13)}=8.939$,
Table 1. Regions activated by Prospect-of-reward, Incompatibility and rTMS

\begin{tabular}{|c|c|c|c|c|c|}
\hline \multirow[b]{2}{*}{ Neural region } & \multirow[b]{2}{*}{ Side } & \multicolumn{3}{|c|}{ MNI coordinates } & \multirow{2}{*}{$\begin{array}{l}\operatorname{Max} \\
z \text {-score }\end{array}$} \\
\hline & & $x$ & y & $z$ & \\
\hline \multirow{3}{*}{\multicolumn{6}{|c|}{$\begin{array}{l}\text { Main effect of Prospect-of-reward } \\
\text { (Prospect-of-reward vs } \\
\text { no-reward) }\end{array}$}} \\
\hline & & & & & \\
\hline & & & & & \\
\hline Cerebellum & Right & 26 & -44 & -28 & 6.02 \\
\hline Cerebellum & Left & -30 & -40 & -32 & 5.78 \\
\hline Caudate & Right & 20 & 10 & 16 & 4.98 \\
\hline Putamen & Left & -26 & -4 & -10 & 4.71 \\
\hline Occipital cortex & Right & 38 & -84 & 2 & 4.41 \\
\hline Putamen & Right & 20 & 10 & 4 & 3.94 \\
\hline Pallidum & Right & 20 & 12 & 0 & 3.87 \\
\hline SN/STN & Right & 16 & -8 & -10 & 3.53 \\
\hline Caudate & Left & -20 & 12 & 18 & 3.29 \\
\hline \multicolumn{6}{|c|}{ Main effect of Incompatibility } \\
\hline \multirow{2}{*}{\multicolumn{6}{|c|}{$\begin{array}{l}\text { (incompatible vs compatible } \\
\text { trials) }\end{array}$}} \\
\hline & & & & & \\
\hline Postcentral gyrus & Left & -42 & -36 & 48 & 5.52 \\
\hline IPC & Left & -52 & -26 & 46 & 4.87 \\
\hline$S M A^{*}$ & Left & -4 & -6 & 56 & 4.40 \\
\hline IPC & Right & 36 & -34 & 36 & 4.16 \\
\hline Cingulate cortex & Left & -8 & 6 & 38 & 3.77 \\
\hline \multirow{2}{*}{\multicolumn{6}{|c|}{$\begin{array}{l}\text { Main effect of rTMS (real vs } \\
\text { sham rTMS) }\end{array}$}} \\
\hline & & & & & \\
\hline - & - & - & - & - & - \\
\hline
\end{tabular}

Results are shown at a threshold of $p \leq 0.05$ familywise error (FWE) corrected at the cluster level. SN, substantia nigra; IPC, inferior parietal cortex. *SMA activation peaked at $y=-6$, but also extended into the pre-SMA. There was no significant main effect of rTMS even at a lower threshold of $P_{\text {uncorrected }} \leq 0.001$.

$p=0.01$; Fig. $1 c)$. There was a statistical trend of Prospect-ofreward $\left(F_{(1,13)}=3.891, p=0.07\right)$, but no significant effects of Response-speed $\left(F_{(1,13)}=1.131, p=0.307\right)$ or $\operatorname{rTMS}\left(F_{(1,13)}=\right.$ $0.034, p=0.856)$.

Since Response-speed modulated the crucial interaction between the effects of rTMS and Prospect-of-reward, $F_{(1,13)}=$ $4.709, p=0.049$, we also analyzed accuracy data separately for fast and slow responses. There was an interaction between rTMS and Prospect-of-reward $\left(F_{(1,13)}=4.711, p=0.049\right)$ and a statistical trend for the interaction rTMS $\times$ Prospect-of-reward $\times$ Incompatibility $\left(F_{(1,13)}=4.377, p=0.057\right)$ during fast responses, but not during slow responses (respectively, $p=0.168$ and $p=$ 0.978). Post hoc comparison revealed that this interaction during fast responses was driven by a reward-related increase in accuracy rates after real rTMS compared with sham rTMS in incompatible trials $(t=2.249, p=0.03$, two-tailed $t$ test), with accuracy rates increasing from 94.7 to $97.9 \%$ after real rTMS (Fig. 1c, gray box). This improvement was not correlated with rTMS-induced changes in the reward-effect on RTs $(p=0.409)$ and can therefore not merely be explained by an increase in response caution or general effects of rTMS on response speed. Thus, prospect of reward led to improved response speed both after real and sham rTMS. However, prospect of reward only led to improved motor control (as indexed by accuracy rates during fast responses) after real rTMS of the pre-SMA.

\section{Neural activity}

In a first step, we computed the main effects of Prospect-ofreward, Incompatibility, and rTMS, which are listed in Table 1. Prospect of reward induced activation in the bilateral basal ganglia (BG), bilateral cerebellum, and the visual cortex. Response conflict during incompatible trials was linked to activation of a frontoparietal network comprising left SMA extending to the 
pre-SMA, left cingulate cortex, bilateral inferior parietal cortex, and left postcentral gyrus. There were no main effects of rTMS.

Then, to specifically assess the neural mechanisms underlying the behavioral improvement in action control, we applied fMRI covariance analysis using the reward-related benefit in accuracy as our covariance of interest. The analysis revealed that activity in left IFG (peak activation in MNI space: -58 22 4) and left STN (peak activation in MNI space: -14 $-12-6)$ was predicted by individual differences in the rTMS-dependent rewardrelated benefit in accuracy (Fig. 2). The higher the benefit in motor control, the stronger both areas were activated. Of note, this was only significant for the left hemisphere in line with a lateralization of motor control to the contralateral side of the executed movements. This result confirmed that pre-SMA, left IFG, and left STN are critical nodes in a neural network mediating motor control, which we aimed to study in more detail in the final step of the analysis.

\section{Neural connectivity}

To study the functional interplay between pre-SMA, left IFG. and left STN and to assess whether the improvement in motor control was mediated by adaptive changes in neural connectivity we used DCM of effective connectivity (Friston et al., 2003). $\beta$-Estimates and $t$ scores for the main effects in the modeled regions are shown in Table 2. The DCM analysis comprised two steps. First, several models with different connectivity profiles between pre-SMA, left IFG, and left STN were created (Fig. $3 a$ ) and compared using BMS (Penny et al., 2004). BMS can be used to identify the model that best explains the data taking into account model complexity. Second, the connectivity parameters of the best model were extracted to test whether adaptive changes within our network of interest improved predictions of the observed benefit in motor control.

The network model that best explained the data postulated modulatory effects of both Prospect-of-reward and rTMS on the connection from left IFG to left STN (Fig. 3b, model 9). According to differences in the free energy approximation to the logmodel evidence, there was strong evidence for this model $(\Delta F=$ 40 between the best model and second best model (model 3)) resulting in a posterior model probability of $\sim 1$. We therefore based further analysis of network parameters on model 9. This model explained $19.3 \%$ variance of IFG activity, $14.2 \%$ variance of pre-SMA activity, and 17\% variance of STN activity. No "flatline-fits" were present in any of the participants. Coupling parameters (A-matrix and B-matrix) of model 9 are illustrated in Figure $3 c$.

Including modulation of effective connectivity (Prospect-ofreward and rTMS) in a hierarchical multiple linear regression analysis significantly improved predictions of the behavioral benefit in motor control compared with a regression model containing only modulation of neural activity in IFG and STN based on
Table 2. $t$-scores and $\boldsymbol{\beta}$-estimates of main effects in modeled regions

\begin{tabular}{llll}
\hline & Prospect-of-reward & Incompatibility & TMS \\
\hline Pre-SMA & $4.91(+1.5)$ & $4.87(+1.2)$ & $2.90(-1.5)$ \\
IFG & $4.72(+1.5)$ & $4.11(+1.2)$ & $2.14(+1.6)$ \\
STN & $3.44(+0.4)$ & $5.18(+0.5)$ & $2.11(+0.9)$ \\
\hline
\end{tabular}

The $t$-scores reflect the effects of the respective conditions in a random-effects analysis comprising data of all participants (one-sample $t$ test). $\beta$-Estimates are given in parentheses.

the fMRI covariance analysis $\left(R^{2}\right.$ change $\left.=0.124, p=0.036\right)$. Together, the model including modulation of neural activity and connectivity of IFG and STN predicted $>88 \%$ variance $\left(R^{2}=\right.$ 0.886 , adjusted $\left.R^{2}=0.835, p<0.001\right)$, which is illustrated in Figure 4.

\section{Discussion}

Combining off-line rTMS of pre-SMA with fMRI, we assessed how corticosubthalamic motor control networks mediate inhibition of inappropriate response tendencies induced by conflicting stimulus features. Noninvasive brain stimulation and prospect of reward for fast and accurate responses improved control over impulsive response tendencies. This effect was mediated by modulation of IFG-STN activity and connectivity reflecting network reorganization remote to the site of stimulation. We discuss these findings in the context of theories of action control.

Dual-process models have been developed in an attempt to explain the mechanisms underlying the resolution of decision conflict induced by competing response tendencies (Kornblum et al., 1990). According to these models an automatic uncondi- 

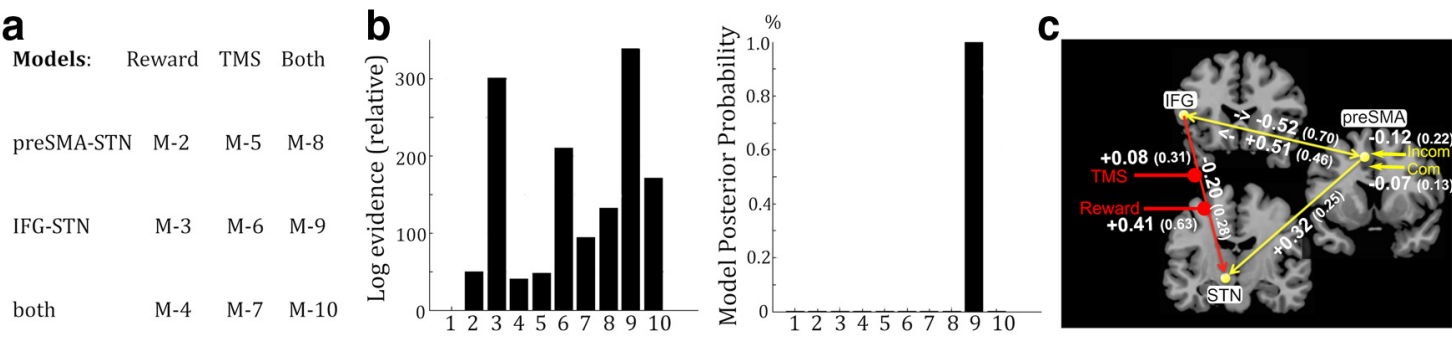

Models

Figure 3. BMS. $\boldsymbol{a}$, Models that were compared using BMS. The models differed with regards to the modulated connection (pre-SMA-STN, IFG—STN, or both) and the experimental modulator (Prospect-of-reward, TMS, or both). We also created a null model (M-1), which postulated that no connections were modulated. The models did not differ with regards to endogenous coupling (A-matrix) or driving inputs (C-matrix; set to pre-SMA). $\boldsymbol{b}$, Comparison of all considered models. There was strong evidence for the model postulating that both Prospect-of-reward and TMS modulated the connection from IFG to STN (model 9). c, Model parameters. Endogenous coupling (A-matrix) is indicated by yellow lines connecting the different regions. Contextual modulation (B-matrix; effect of Reward and TMS) is indicated by red lines modulating IFG-STN. The yellow arrow entering the network via pre-SMA indicates the driving inputs (compatible and incompatible trials). Coupling values are given in mean (SD).

a

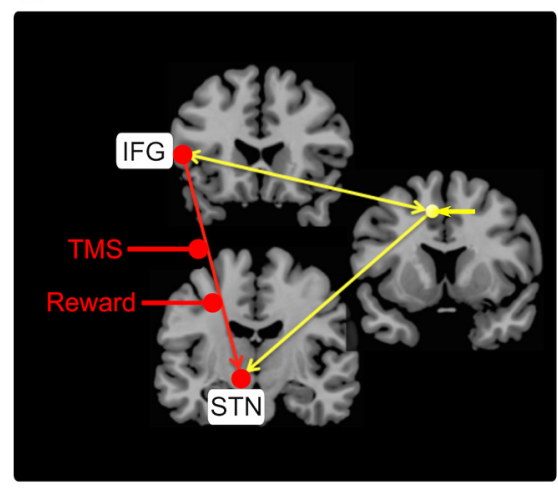

b

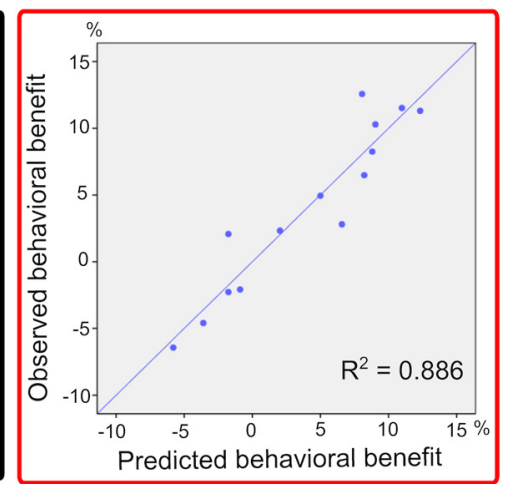

Figure 4. Hierarchical multiple linear regression analysis. $\boldsymbol{a}$, Illustration of the predictors. The original regression model included modulation of neural activity in IFG and STN as observed in the covariance-based fMRI analysis $\left(R^{2}=0.762\right)$. Entering connectivity values from the DCM analysis reflecting modulation of coupling by Prospect-of-reward and TMS (B-matrix) significantly improved model predictions of the behavioral benefit (reward-related benefit in accuracy after rTMS) with an $R^{2}$-change of $0.124(p=0.036)$. The independent variables (predictors) of the regression model are marked in red. $\boldsymbol{b}$, Regression model predictions. The regression model comprising modulation of activity and connectivity of the IFG-STN pathway predicted $>88 \%$ variance of the behavioral benefit $(p<0.001)$.

tional response tendency elicited by the (irrelevant) spatial position of the stimulus has to be inhibited when it is in conflict with the relevant stimulus dimension (here color). Since the automatic response is processed more quickly than the appropriate response, subjects have to slow down their motor responses and "hold their horses" when a conflict between response tendencies has been detected (Ridderinkhof, 2002; Frank et al., 2007). If subjects fail to do so, the impulsive response might be committed through the motor system, resulting in an erroneous and rapid response.

In the current study, we only observed a behavioral effect of rTMS for between-trials conflict according to feature integration theory (Hommel et al., 2004), but not for the classical within-trial conflict (Simon, 1967). One possible explanation is that the behavioral effects of between-trials conflict on RT and accuracy rates were more pronounced than the corresponding within-trial conflict. Thus, performance on Corresponding and Noncorresponding trials might have been too similar to allow a differential modulation by rTMS. On the other hand, a recent study has shown that the preSMA is particularly important in resolving response conflict between trials, i.e., when a Noncorresponding trial follows a Corresponding trial in the flanker task (Soutschek et al., 2013). This view is in line with studies demonstrating a central role of pre-SMA in switching stimulusresponse rules between trials (Rushworth et al., 2002; Neubert et al., 2010) and is also compatible with our observations.
Neurobiologically, it has been suggested that the BG are key in controlling inappropriate response tendencies (Nambu et al., 2002; Ridderinkhof et al., 2011). Within the BG, the STN is optimally positioned within the network for stopping impulsive responses, since it receives direct afferents from higher order cortical regions in a hyperdirect pathway, allowing a rapid activation of STN by cortical regions (Nambu et al., 2002; Magill et al., 2004). Via diverging axons to the pallidum, STN activation leads to a broad inhibition of thalamocortical projections resulting in a global movement inhibition (Aron, 2011). Studies have shown that STN activation coincides with activation of pre-SMA and IFG during response conflict and movement inhibition (Aron et al., 2007; Forstmann et al., 2008). Since both pre-SMA and IFG have a hyperdirect pathway to the STN (Aron et al., 2007), it was proposed that pre-SMA, IFG, and STN constitute a neural network controlling inappropriate responses (Aron et al., 2007; Ridderinkhof et al., 2011). This was supported by studies showing that deep-brain stimulation of the STN in patients with Parkinson's disease disrupts pre-SMA activation and behavioral decision (Cavanagh et al., 2011) and motor thresholds (Ballanger et al., 2009; Wylie et al., 2010), whereas it strengthens IFG activation and behavioral inhibitory efficiency (van den Wildenberg et al., 2006; Wylie et al., 2010; Swann et al., 2011, 2012). Effective connectivity modeling of stop-related brain activity in healthy individuals shows that connection strengths for the pre-SMA-STN and IFG-STN pathways are opposite in sign (Jahfari et al., 2012). However, there is considerable disagreement about how these regions interact to control inappropriate responses.

On the one hand, it has been suggested that the pre-SMA is the "highest" hub in a hierarchically organized motor control network, which sets inhibitory motor processes into operation when response conflict has been detected. Activity in the preSMA has been found to precede activity in the IFG during action override (Neubert et al., 2010) and action inhibition (Swann et al., 2012). When action tendencies conflict, the pre-SMA may send rapid signals to the BG (Hikosaka and Isoda, 2010) to increase response thresholds (Forstmann et al., 
2010) while recruiting the IFG to suppress and override the inappropriate urge.

On the other hand, a parallel network model of the pre-SMA-STN and IFG-STN pathway proposes that the activation of the IFG-STN connection during response conflictthough not independent from pre-SMA function-does not critically depend on pre-SMA activation and might thus become particularly valuable when pre-SMA is insufficient in controlling erroneous responses. For instance, trial-by-trial variability in IFG-STN interaction may be complemented through compensatory recruitment of pre-SMA-STN interaction (Wiecki and Frank, 2010).

The results of the current study provide causal evidence for a parallel organization of the pre-SMA-STN and IFG-STN pathways, since improved control over impulsive responses was linked to an enhanced IFG-STN connectivity after pre-SMA had been disrupted. The hierarchical model, which proposes that pre-SMA activates the IFG-STN pathway to override the inappropriate response, would have predicted the opposite effect, which we did not observe, namely impaired control over impulsive responses and impaired IFG-STN connectivity after inhibitory stimulation of pre-SMA.

Combining rTMS and positron emission tomography, Obeso et al. (2013) have recently demonstrated that stimulation of preSMA can improve inhibitory efficiency in a Go-No-go task, which was accompanied by increased activity of left IFG. These results are in strong agreement with our study and provide additional evidence for a parallel organization of pre-SMA and IFG.

The left lateralization of the detected network seems at odds with previous studies relating inhibition to a right-lateralized network (Aron, 2011). However, recent evidence suggests that frontoparietal regions of the left hemisphere mediate inhibitory efficiency (Hirose et al., 2012), which is in line with our findings demonstrating that improvement of inhibitory control is related to activity of left IFG and STN.

Importantly, the improvement in performance after rTMS was present only during prospect of reward for fast and accurate responses, suggesting an interaction between the level of motivation and motor control. The neural mechanisms mediating motivational modulation of motor control in this context are not well understood. However, a central role in this process has been assigned to the BG (Mogenson et al., 1980), since they comprise neural loops processing information not only from motor but also from limbic regions (Alexander et al., 1986). These loops are to some extent spatially segregated, but also interact within the BG (Haber et al., 2006; Belin and Everitt, 2008). In line with this, we found that prospect of reward induced activity in the BG and modulated IFG-STN connectivity.

Additionally, we found bilateral cerebellar activation during prospect of reward. While the cerebellum has traditionally been related to basic motor control, there is increasing evidence that the cerebellum is also involved in modulating "higher cognitive" functions (Ramnani, 2006). Interestingly, recent evidence suggests that the cerebellum is important when effort has to be put into a task (Peterson et al., 2012). In the current task, increased activation of the cerebellum might be related to the increased effort needed to speed up responses to gain a financial reward, although such reverse inference must await future confirmation.

The neural mechanisms underlying changes in neural connectivity by motivational incentives are unclear. However, there is increasing evidence that dopamine is central to reward-related modulation of neural connectivity, e.g., via dopamine-dependent induction of long-term potentiation (LTP; Calabresi et al., 2007). Dopaminedependent modulation of connectivity is central to reinforcement learning (Redgrave et al., 2010) and motivational modulation of behavior (Robbins and Everitt, 2007). Importantly, it has recently been shown that the interface between motivation and cognitive control is mediated by striatal dopamine release (Aarts et al., 2012, 2011) and modulation of corticostriatal connectivity (Harsay et al., 2011). During motivational learning dopamine release increases activity of D1-type receptor-related movement-facilitating corticostriatal pathways and decreases activity of D2-type receptor-related movement-inhibiting pathways, thus reinforcing motor programs, which have led to a rewarding outcome (Frank et al., 2004, 2007). This mechanism allows adjusting behavior to action-specific outcomes and motivational context. Since dopamine-dependent induction of LTP also takes place in extrastriatal nuclei of the BG (Prescott et al., 2009), such a mechanism might underlie the observed increase in connectivity from IFG to STN in the current study.

Our proof of principle study has important implications for experimental modulation of the pre-SMA-IFG-STN network: while disruption of pre-SMA through rTMS did not have any main effects on motor control, combining rTMS with explicit financial incentives enabled an improvement of motor control in healthy subjects. Given the variable effects of rTMS (Hamada et al., 2013) and the limited sample size $(n=14)$ future research, in particular studies with larger sample sizes, are needed to confirm and further explore the results observed in this study.

In summary, we provide causal evidence for a pivotal role of the IFG-STN pathway during control of inappropriate responses and demonstrate that motor control can be modified by shaping corticosubthalamic connectivity when combining motivational incentives and noninvasive brain stimulation.

\section{References}

Aarts E, van Holstein M, Cools R (2011) Striatal dopamine and the interface between motivation and cognition. Front Psychol 2:163. CrossRef Medline

Aarts E, Helmich RC, Janssen MJ, Oyen WJ, Bloem BR, Cools R (2012) Aberrant reward processing in Parkinson's disease is associated with dopamine cell loss. Neuroimage 59:3339-3346. CrossRef Medline

Alexander GE, DeLong MR, Strick PL (1986) Parallel organization of functionally segregated circuits linking basal ganglia and cortex. Annu Rev Neurosci 9:357-381. CrossRef Medline

Aron AR (2011) From reactive to proactive and selective control: developing a richer model for stopping inappropriate responses. Biol Psychiatry 69:e55-68. CrossRef Medline

Aron AR, Behrens TE, Smith S, Frank MJ, Poldrack RA (2007) Triangulating a cognitive control network using diffusion-weighted magnetic resonance imaging (MRI) and functional MRI. J Neurosci 27:3743-3752. CrossRef Medline

Ballanger B, van Eimeren T, Moro E, Lozano AM, Hamani C, Boulinguez P, Pellecchia G, Houle S, Poon YY, Lang AE, Strafella AP (2009) Stimulation of the subthalamic nucleus and impulsivity: release your horses. Ann Neurol 66:817-824. CrossRef Medline

Belin D, Everitt BJ (2008) Cocaine seeking habits depend upon dopaminedependent serial connectivity linking the ventral with the dorsal striatum. Neuron 57:432-441. CrossRef Medline

Calabresi P, Picconi B, Tozzi A, Di Filippo M (2007) Dopamine-mediated regulation of corticostriatal synaptic plasticity. Trends Neurosci 30:211219. CrossRef Medline

Cavanagh JF, Wiecki TV, Cohen MX, Figueroa CM, Samanta J, Sherman SJ, Frank MJ (2011) Subthalamic nucleus stimulation reverses mediofrontal influence over decision threshold. Nat Neurosci 14:1462-1467. CrossRef Medline

Forstmann BU, van den Wildenberg WP, Ridderinkhof KR (2008) Neural mechanisms, temporal dynamics, and individual differences in interference control. J Cogn Neurosci 20:1854-1865. CrossRef Medline

Forstmann BU, Anwander A, Schäfer A, Neumann J, Brown S, Wagenmakers EJ, Bogacz R, Turner R (2010) Cortico-striatal connections predict control over speed and accuracy in perceptual decision making. Proc Natl Acad Sci U S A 107:15916-15920. CrossRef Medline 
Frank MJ, Seeberger LC, O’Reilly RC (2004) By carrot or by stick: cognitive reinforcement learning in parkinsonism. Science 306:1940-1943. CrossRef Medline

Frank MJ, Samanta J, Moustafa AA, Sherman SJ (2007) Hold your horses: impulsivity, deep brain stimulation, and medication in parkinsonism. Science 318:1309-1312. CrossRef Medline

Friston KJ, Williams S, Howard R, Frackowiak RS, Turner R (1996) Movement-related effects in fMRI time-series. Magn Reson Med 35:346355. CrossRef Medline

Friston KJ, Harrison L, Penny W (2003) Dynamic causal modelling. Neuroimage 19:1273-1302. CrossRef Medline

Friston K, Mattout J, Trujillo-Barreto N, Ashburner J, Penny W (2007) Variational free energy and the Laplace approximation. Neuroimage 34: 220-234. CrossRef Medline

Haber SN, Kim KS, Mailly P, Calzavara R (2006) Reward-related cortical inputs define a large striatal region in primates that interface with associative cortical connections, providing a substrate for incentive-based learning. J Neurosci 26:8368-8376. CrossRef Medline

Hamada M, Murase N, Hasan A, Balaratnam M, Rothwell JC (2013) The role of interneuron networks in driving human motor cortical plasticity. Cereb Cortex 23:1593-1605. CrossRef Medline

Harsay HA, Cohen MX, Oosterhof NN, Forstmann BU, Mars RB, Ridderinkhof KR (2011) Functional connectivity of the striatum links motivation to action control in humans. J Neurosci 31:10701-10711. CrossRef Medline

Hikosaka O, Isoda M (2010) Switching from automatic to controlled behavior: cortico-basal ganglia mechanisms. Trends Cogn Sci 14:154-161. CrossRef Medline

Hirose S, Chikazoe J, Watanabe T, Jimura K, Kunimatsu A, Abe O, Ohtomo K, Miyashita Y, Konishi S (2012) Efficiency of go/no-go task performance implemented in the left hemisphere. J Neurosci 32:9059-9065. CrossRef Medline

Hommel B, Proctor RW, Vu KP (2004) A feature-integration account of sequential effects in the Simon task. Psychol Res 68:1-17. CrossRef Medline

Jahfari S, Verbruggen F, Frank MJ, Waldorp LJ, Colzato L, Ridderinkhof KR, Forstmann BU (2012) How preparation changes the need for top-down control of the basal ganglia when inhibiting premature actions. J Neurosci 32:10870-10878. CrossRef Medline

Johansen-Berg H, Behrens TE, Robson MD, Drobnjak I, Rushworth MF, Brady JM, Smith SM, Higham DJ, Matthews PM (2004) Changes in connectivity profiles define functionally distinct regions in human medial frontal cortex. Proc Natl Acad Sci U S A 101:13335-13340. CrossRef Medline

Kornblum S, Hasbroucq T, Osman A (1990) Dimensional overlap: cognitive basis for stimulus-response compatibility-a model and taxonomy. Psychol Rev 97:253-270. CrossRef Medline

Kosslyn SM, Cacioppo JT, Davidson RJ, Hugdahl K, Lovallo WR, Spiegel D, Rose R (2002) Bridging psychology and biology. The analysis of individuals in groups. Am Psychol 57:341-351. CrossRef Medline

Lambert C, Zrinzo L, Nagy Z, Lutti A, Hariz M, Foltynie T, Draganski B, Ashburner J, Frackowiak R (2012) Confirmation of functional zones within the human subthalamic nucleus: patterns of connectivity and subparcellation using diffusion weighted imaging. Neuroimage 60:83-94. CrossRef Medline

Lucerna S, Salpietro FM, Alafaci C, Tomasello F (2002) In vivo atlas of deep brain structures, Vol 1. Berlin: Springer.

Lund TE, Madsen KH, Sidaros K, Luo WL, Nichols TE (2006) Non-white noise in fMRI: does modelling have an impact? Neuroimage 29:54-66. CrossRef Medline

Magill PJ, Sharott A, Bevan MD, Brown P, Bolam JP (2004) Synchronous unit activity and local field potentials evoked in the subthalamic nucleus by cortical stimulation. J Neurophysiol 92:700-714. CrossRef Medline

Mogenson GJ, Jones DL, Yim CY (1980) From motivation to action: functional interface between the limbic system and the motor system. Prog Neurobiol 14:69-97. CrossRef Medline

Monakow KH, Akert K, Künzle H (1978) Projections of the precentral motor cortex and other cortical areas of the frontal lobe to the subthalamic nucleus in the monkey. Exp Brain Res 33:395-403. Medline

Nambu A, Tokuno H, Takada M (2002) Functional significance of the cortico-subthalamo-pallidal 'hyperdirect' pathway. Neurosci Res 43:111117. CrossRef Medline
Neubert FX, Mars RB, Buch ER, Olivier E, Rushworth MF (2010) Cortical and subcortical interactions during action reprogramming and their related white matter pathways. Proc Natl Acad Sci U S A 107:13240-13245. CrossRef Medline

Obeso I, Cho SS, Antonelli F, Houle S, Jahanshahi M, Ko JH, Strafella AP (2013) Stimulation of the pre-SMA influences cerebral blood flow in frontal areas involved with inhibitory control of action. Brain Stimul 6:769-776. CrossRef Medline

Oldfield RC (1971) The assessment and analysis of handedness: the Edinburgh inventory. Neuropsychologia 9:97-113. CrossRef Medline

Penny WD, Stephan KE, Mechelli A, Friston KJ (2004) Comparing dynamic causal models. Neuroimage 22:1157-1172. CrossRef Medline

Peterson TC, Villatoro L, Arneson T, Ahuja B, Voss S, Swain RA (2012) Behavior modification after inactivation of cerebellar dentate nuclei. Behav Neurosci 126:551-562. CrossRef Medline

Prescott IA, Dostrovsky JO, Moro E, Hodaie M, Lozano AM, Hutchison WD (2009) Levodopa enhances synaptic plasticity in the substantia nigra pars reticulata of Parkinson's disease patients. Brain 132:309-318. CrossRef Medline

Ramnani N (2006) The primate cortico-cerebellar system: anatomy and function. Nat Rev Neurosci 7:511-522. CrossRef Medline

Redgrave P, Rodriguez M, Smith Y, Rodriguez-Oroz MC, Lehericy S, Bergman H, Agid Y, DeLong MR, Obeso JA (2010) Goal-directed and habitual control in the basal ganglia: implications for Parkinson's disease. Nat Rev Neurosci 11:760-772. CrossRef Medline

Ridderinkhof KR (2002) Micro- and macro-adjustments of task set: activation and suppression in conflict tasks. Psychol Res 66:312-323. CrossRef Medline

Ridderinkhof KR, Forstmann BU, Wylie SA, Burle B, van den Wildenberg WPM (2011) Neurocognitive mechanisms of action control: resisting the call of the Sirens. WIREs Cogn Sci 2:174-192. CrossRef

Robbins TW, Everitt BJ (2007) A role for mesencephalic dopamine in activation: commentary on Berridge (2006). Psychopharmacology 191:433_ 437. CrossRef Medline

Rubia K, Russell T, Overmeyer S, Brammer MJ, Bullmore ET, Sharma T, Simmons A, Williams SC, Giampietro V, Andrew CM, Taylor E (2001) Mapping motor inhibition: conjunctive brain activations across different versions of go/no-go and stop tasks. Neuroimage 13:250-261. CrossRef Medline

Rushworth MF, Hadland KA, Paus T, Sipila PK (2002) Role of the human medial frontal cortex in task switching: a combined fMRI and TMS study. J Neurophysiol 87:2577-2592. Medline

Siebner HR, Rothwell J (2003) Transcranial magnetic stimulation: new insights into representational cortical plasticity. Exp Brain Res 148:1-16. CrossRef Medline

Simon JR (1967) Ear preference in a simple reaction-time task. J Exp Psychol 75:49-55. CrossRef Medline

Soutschek A, Taylor PC, Müller HJ, Schubert T (2013) Dissociable networks control conflict during perception and response selection: a transcranial magnetic stimulation Study. J Neurosci 33:5647-5654. CrossRef Medline

Swann NC, Cai W, Conner CR, Pieters TA, Claffey MP, George JS, Aron AR, Tandon N (2012) Roles for the pre-supplementary motor area and the right inferior frontal gyrus in stopping action: electrophysiological responses and functional and structural connectivity. Neuroimage 59: 2860-2870. CrossRef Medline

Swann N, Poizner H, Houser M, Gould S, Greenhouse I, Cai W, Strunk J, George J, Aron AR (2011) Deep brain stimulation of the subthalamic nucleus alters the cortical profile of response inhibition in the beta frequency band: a scalp EEG study in Parkinson's disease. J Neurosci 31: 5721-5729. CrossRef Medline

van den Wildenberg WP, van Boxtel GJ, van der Molen MW, Bosch DA, Speelman JD, Brunia CH (2006) Stimulation of the subthalamic region facilitates the selection and inhibition of motor responses in Parkinson's disease. J Cogn Neurosci 18:626-636. CrossRef Medline

Wiecki TV, Frank MJ (2010) Neurocomputational models of motor and cognitive deficits in Parkinson's disease. Prog Brain Res 183:275-297. CrossRef Medline

Wylie SA, Ridderinkhof KR, Elias WJ, Frysinger RC, Bashore TR, Downs KE, van Wouwe NC, van den Wildenberg WP (2010) Subthalamic nucleus stimulation influences expression and suppression of impulsive behaviour in Parkinson's disease. Brain 133:3611-3624. CrossRef Medline 